\title{
Influence of Pregnancy on Neuromyelitis Optica From an Ophthalmological Point of View: a Case Report and Literature Review
}

Fatih Aslan ( $\sim$ fatih.aslan@alanya.edu.tr)

Alaaddin Keykubat University Alanya Education and Research Hospital, Department of Ophthalmology https://orcid.org/0000-0001-6019-2815

Meral Tuğba-Çimşir

Alaaddin Keykubat University Alanya Education and Research Hospital, Department of Obstetrics and Gynecology https://orcid.org/0000-0002-9738-3233

Ece Özdemir-Öktem

Alaaddin Keykubat University Alanya Education and Research Hospital, Department of Neurology https://orcid.org/0000-0002-1264-5696

\section{Case Report}

Keywords: Neuromyelitis optica, optic neuritis, pregnancy, optical coherence tomography

Posted Date: October 12th, 2021

DOl: https://doi.org/10.21203/rs.3.rs-964025/v1

License: (c) (1) This work is licensed under a Creative Commons Attribution 4.0 International License. Read Full License 


\section{Abstract}

Neuromyelitis Optica (NMO, Devic's disease) is a rare demyelinating disease of the central nervous system leading to optic neuritis and transverse myelitis. The clinical course of the disease and ocular changes in pregnancy is still not well known. Here, we present the subclinical ophthalmological changes which are evaluated by spectral domain optical coherence tomography (SD-OCT) during the 39 weeks gestation and postpartum period in a pregnant woman with a diagnosis of NMO. Additionally, we present the obstetric and neurologic course of our patient and review the literature. A 30-year-old female with a history of NMO had been ophthalmologically examined and performed SD-OCT every trimester periodically to observe the effects of pregnancy on the disease course. No ophthalmological changes were observed during the pregnancy and postpartum period. Cesarean delivery was preferred due to obstetric indication. The patient was discharged on the second day of postpartum period.

\section{Introduction}

Neuromyelitis optica (NMO) or Devic's disease is an autoimmune inflammatory disease of the central nervous system (CNS) that predominantly affects the brainstem, spinal cord and optic nerves [1]. This severe disease is characterized by optic neuritis and transverse myelitis, which may lead to paralysis and blindness [2]. The presence of pathogenic antibodies against the CNS water channel aquaporin-4 (AQP4) is responsible for NMO and mostly found in the serum of patients with NMO [3]. Additionally, the appearance of spinal cord MRI, which is characterized with a T2-weighted lesion that contiguously extends over 3 or more vertebral segments represents the most useful diagnostic for NMO. This autoantibody-induced disease principally affects women, many of whom are of childbearing age [4].

Although few data exist in pregnant patients with NMO, most of the studies indicate that pregnancies may be associated with increased disease activity and more severe disability both pregnancy and postpartum period. Unlike MS, relapse rate of NMO is not reduced during the trimesters and some studies have found higher risks of pregnancy complications, e.g., miscarriages and pre-eclampsia [5].

In 2015, diagnostic criteria of NMO have been revised using core clinical features, serology status of AQP4 and imaging features [2]. Optic neuritis $(\mathrm{ON})$, one of the core criteria of $\mathrm{NMO}$, is a common condition involving inflammation, demyelination, and axonal injury in the optic nerve. This process may lead to retinal ganglion cell (RGC) death, decreased macular volume, and visual dysfunction or permanent visual loss [6]. Spectral-domain optical coherence tomography (SD-OCT), which is a noninvasive imaging tool can provide data on peripapillary and macular RNFL thicknesses and generate macular maps [7].

To our knowledge, until now, it has not been published a case report that demonstrated the subclinical and clinical ophthalmological changes in a pregnant patient with NMO during the gestational period. Here we report the periodical ophthalmological examination findings of 27-year-old pregnant women with the diagnosis of NMO. 


\section{Case Report}

A 27-year-old female patient was admitted to our Obstetrics and Gynecology Department with menstrual delay and suspicion of pregnancy. Suprapubic pelvic ultrasonography revealed a $10-\mathrm{mm}$ gestational sac consistent with 5-week pregnancy.

In her medical history, she had a retrobulbar neuritis presenting with low vision and visual blurring at the age of 15. Brain magnetic imaging was normal except bilateral hyperintensities in the optic tractus which is consisted with optic neuritis. In the spinal MRI, there was a hyperintense lesion without contrast enhancement in the cervical cord at the level of C2-C5. CSF analyses were normal and, no oligoclonal bands were observed. In visual evoked potential (VEP) examination, there were prolonged p100 latanses. Aquaporin-4 antibodies were negative. With these findings' patient was diagnosed with seronegative $\mathrm{NMO}$ and had been under periodic follow-up for 15 years. At admission to our clinic, she was not receiving any prophylactic or immunomodulator treatment. Routine laboratory tests were normal.

Fetal development was frequently monitored, and obstetric ultrasounds were all normal. Double and triple antenatal screening tests including PAPP-A: $11.6 \mathrm{~m} \mathrm{IU} / \mathrm{Ml}$, f $\beta$-Hcg: $68.5 \mathrm{ng} / \mathrm{Ml}$, NT MOM: 0.79, AFP: 45.7 $\mathrm{IU} / \mathrm{mL}$ and E3: $2.11 \mathrm{ng} / \mathrm{mL}$ were normal. There was no abnormality in her neurological examination and no changes were observed during the 39 weeks of gestation. In her spinal MRI examination, which is performed in the gestational period; a weak hyperintense appearance was detected in the T2-weighted series at the level of the C2-C4 vertebra in the cervical spinal cord (Fig. 1). Apart from this lesion, there was no pathological finding or contrast enhancement in the brain and other spinal cord regions.

During pregnancy, she underwent a detailed ophthalmologic examination periodically including best corrected visual acuity measurement using Snellen chart, intraocular pressure (IOP) measurement using Goldmann applanation tonometry, and slit-lamp, dilated fundus examination, OCT measurements (RTVue-XR Avanti, Optovue Inc., Fremont, CA, USA) in the ophthalmology outpatient clinic. The fundus examination was performed at regular intervals and the OCT measurements was extracted. In the first examination of the case after pregnancy detection; Her visual acuity was counting fingers at 1 meter in both eyes, marked optic disc pallor and atrophy in both eyes (Fig. 2), peripapillary nerve fiber thickness (RNFL) was $50 \mu \mathrm{m}$ and $43 \mu \mathrm{m}$ in the right and left eyes, and ganglion cell complex thickness (GCC) was $52 \mu \mathrm{m}$ and $54 \mu \mathrm{m}$ in the right and left eyes, respectively. Central retinal thickness (CRT) was $225 \mu \mathrm{m}$ and $221 \mu \mathrm{m}$ in the right and left eyes, respectively. Intraocular pressure was $13 \mathrm{mmHg}$ in the right and left eyes. The patient was not receiving anti-glaucomatous therapy. Visual field test could not be performed due to low vision level. RNFL thickness in the right and left eyes at the end of the first trimester, second trimester and third trimester; $50 \mu \mathrm{m}, 46 \mu \mathrm{m} ; 50 \mu \mathrm{m}, 46 \mu \mathrm{m} ; 48 \mu \mathrm{m}, 46 \mu \mathrm{m}$ found, respectively. GCC thickness in the right and left eyes at the end of the first trimester, second trimester and third trimester; $53 \mu \mathrm{m}, 54 \mu \mathrm{m} ; 56 \mu \mathrm{m}, 54 \mu \mathrm{m} ; 54 \mu \mathrm{m}, 55 \mu \mathrm{m}$ found, respectively. In addition, CRT values in the right and left eyes at the end of the 1st, 2nd and 3rd trimesters; $223 \mu \mathrm{m}, 215 \mu \mathrm{m} ; 223 \mu \mathrm{m}, 217 \mu \mathrm{m} ; 226 \mu \mathrm{m}$ and $223 \mu \mathrm{m}$ were found, respectively. At the end of the third trimester, intraocular pressure was measured as $10 \mathrm{mmHg}$ 
and $11 \mathrm{mmHg}$ on the right and left, respectively. During the pregnancy period no changes were observed in visual acuity.

As gestation reached 39 weeks, the patient was hospitalized because of regular uterine contractions. Although the normal vaginal delivery was planned, an emergency cesarean section was performed under general anesthesia because of cephalopelvic disproportion (CPD). No complications had occurred during the delivery and postpartum period.

\section{Discussion}

We report the ophthalmological course and subclinical ocular changes of a 21-year-old pregnant woman with a 12-year history of NMO. There is poor knowledge about the influence of pregnancy in NMO. To our knowledge, there is no data about the periodical ophthalmological examination of patients with NMO during pregnancy.

Several studies have indicated that pregnancy negatively influences NMO disease course, due to the increased relapse rate during the gestational period [8-10]. In an international cohort study including 85 pregnancies, it has been claimed that NMO may be a risk factor for other pregnancy complications such as miscarriage and pre-eclampsia. The miscarriage rate was found $12.9 \%, 7$ were in the first trimester, 1 in the second trimester, and 3 at an unknown time within the first 24 weeks. Indeed, preeclampsia rate was higher among pregnant patients with NMO compared with the general population (116.5\% vs $3.2 \%$, respectively [11]. Wang et al. reported some neonatal malformations that might be related to maternal NMO, including undeveloped external ear, lacrimal obstruction, and scoliosis. Pre-eclampsia was not observed during the pregnancy in this cohort study [12]. In this case, we did not observe any relapses or pregnancy complications due to the NMO. Furthermore, during the delivery and postpartum period, no complications or neonatal malformations have been observed in our patient with NMO.

From the immunological perspective, changes during pregnancy and the disease-worsening factors are still unclear. Very few studies have provided data on the role of immunological factors. In addition to the expression in astrocytes, recent reports have reported the presence of AQP4 in human and animal placenta. The expression by placental syncytiotrophoblast leading to an AQP4-antibody mediated attack on the placenta may be responsible for miscarriages due to the occurrence of placentitis [9]. Another disease-worsening factor in pregnancy may be sexual hormones. Increase of estrogen levels during pregnancy stimulate immunoglobulin production and influence the inflammatory profile by switching from Th1 (T-helper cell type 1) cytokine response to Th2 (T-helper cell type 2) and Th2 related to humoral immunity predominate during the gestational period. This immunological shift toward humoral immunity may contribute to increased pregnancy complications in patients with $\mathrm{NMO}[9,11,13,14]$.

In a systematic review, it has been reported that maternal symptoms during pregnancy were sensorial abnormalities, neuropathic pain, motor weakness, visual symptoms, incontinence and spasticity [15]. Most of the patients with NMO had an unfavorable relapse with severe vision reduction in the gestational period. In a recent study, it has been shown that early age of onset predicts the severity of visual 
impairment in patients with NMO spectrum disorder [16]. In consistent with this literature, our patient had an early onset of NMO and poor visual outcome.

OCT is an analog of B-mode ultrasonography using infrared light instead of ultrasound to provide data on RNFL thicknesses and macular maps with segmental thicknesses and volumes. With the recent development of SD-OCT, it is possible to evaluate detailed different layers of retina with enhanced resolution $(2 \mu \mathrm{m})$, shorter acquisition times, three-dimensional scans, and video imaging $[7,17]$. Additionally, SD-OCT provides an information about the differential diagnosis of demyelinating optic neuritis. In a prospective case-control study, Zhaou et al. compared the OCT characteristics in ON associated with $\mathrm{NMO}$ and idiopathic ON [18]. They concluded that NMO-ON group had more pronounced RNFL thinning in all quadrants and visual function impairment than the idiopathic ON group. They also reported that microcystic macular edema (MME) was more common in patients with $\mathrm{NMO}(32.2 \%)$ than in those with idiopathic optic neuritis $(10.75 \%)(p=0.001)$ [18]. It has also been reported in previous reports that RNFL and macula were thinner and retinal atrophy is more severe and quick than the other demyelinating diseases such as multiple sclerosis. In addition to these morphological differences; clinically, optic neuritis in NMO has more female preponderance, higher relapse rate, more bilateral involvement and worse visual prognosis compared with other demyelinating diseases $[7,18]$. Several studies showed that the RNFL thickness of patients with NMO is reduced to 55-83 $\mu \mathrm{m}$, compared to 93$108 \mu \mathrm{m}$ in the respective control groups [18-20].

\section{Conclusion}

Although the association of pregnancy and NMO is extremely rare, the few available data indicate an effect on disease exacerbation. Our case may be contribute to literature on how pregnancy affects NMO from the ophthalmological view and observation of subclinical changes on the central retina and peripapillary nerve fiber thickness.

\section{Declarations}

The authors declare no competing interests. Written informed consent of the participant patient was obtained for the publication of this case report and any accompanying images

\section{References}

1. Wingerchuk DM, Weinshenker BG. Neuromyelitis optica: clinical predictors of a relapsing course and survival. Neurology. 2003 Mar;60(5):848-853.

2. Wingerchuk DM, Banwell $B$, Bennett JL, et al. International consensus diagnostic criteria for neuromyelitis optica spectrum disorders. Neurology. $2015 \mathrm{Jul} ; 85(2): 177-189$.

3. Papadopoulos MC, Verkman AS. Aquaporin 4 and neuromyelitis optica. Lancet Neurol. 2012 Jun;11(6):535-544. 
4. Wingerchuk DM, Lennon VA, Lucchinetti CF, Pittock SJ, Weinshenker BG. The spectrum of neuromyelitis optica. Lancet Neurol. 2007 Sep;6(9):805-815.

5. Borisow N, Hellwig K, Paul F. Neuromyelitis optica spectrum disorders and pregnancy: relapsepreventive measures and personalized treatment strategies. EPMA J. 2018 Aug;9(3):249-256.

6. Frohman EM, Frohman TC, Zee DS, McColl R, Galetta S. The neuro-ophthalmology of multiple sclerosis. Lancet Neurol. 2005 Feb;4(2):111-121.

7. Bennett JL, de Seze J, Lana-Peixoto M, et al. Neuromyelitis optica and multiple sclerosis: Seeing differences through optical coherence tomography. Mult Scler. 2015 May;21(6):678-688.

8. Kim W, Kim SH, Nakashima I, et al. Influence of pregnancy on neuromyelitis optica spectrum disorder. Neurology. 2012 Apr;78(16):1264-1267.

9. Klawiter EC, Bove R, Elsone L, et al. High risk of postpartum relapses in neuromyelitis optica spectrum disorder. Neurology. 2017 Nov;89(22):2238-2244.

10. Mao-Draayer $Y$, Thiel S, Mills EA, et al. Neuromyelitis optica spectrum disorders and pregnancy: therapeutic considerations. Nat Rev Neurol. 2020 Mar;16(3):154-170.

11. Nour MM, Nakashima I, Coutinho E, et al. Pregnancy outcomes in aquaporin-4-positive neuromyelitis optica spectrum disorder. Neurology. 2016 Jan;86(1):79-87.

12. Wang L, Zhou L, ZhangBao J et al. Neuromyelitis optica spectrum disorder: pregnancy-related attack and predictive risk factors. J Neurol Neurosurg Psychiatry. 2020 Nov;92(1):53-61.

13. Salvador NRS, Brito MNG, Alvarenga MP, Alvarenga RMP. Neuromyelitis optica and pregnancypuerperal cycle. Mult Scler Relat Disord. 2019 Sep;34:59-62.

14. Davoudi V, Keyhanian K, Bove RM, Chitnis T. Immunology of neuromyelitis optica during pregnancy. Neurol Neuroimmunol Neuroinflamm. 2016 Oct;3(6):e288.

15. D'Souza R, Wuebbolt D, Andrejevic K, et al. Pregnancy and Neuromyelitis Optica Spectrum Disorder Reciprocal Effects and Practical Recommendations: A Systematic Review. Front Neurol. 2020 Oct; $11: 544434$.

16. Macaron G, Khoury J, Bena J, et al. Early age of onset predicts severity of visual impairment in patients with neuromyelitis optica spectrum disorder. Mult Scler. 2021 Jan;e1352458520981736.

17. Bock M, Brandt AU, Dörr J, et al. Time domain and spectral domain optical coherence tomography in multiple sclerosis: A comparative cross-sectional study. Mult Scler. 2010 Jul;16(7):893-896.

18. Zhao X, Qiu W, Zhang Y, et al. A prospective case-control study comparing optical coherence tomography characteristics in neuromyelitis optica spectrum disorder- optic neuritis and idiopathic optic neuritis. BMC Ophthalmol. 2018 Sep;18(1):247.

19. De Seze J, Blanc F, Jeanjean L, et al. Optical coherence tomography in neuromyelitis optica. Arch Neurol. 2008 Jul;65(7):920-923.

20. Nakamura M, Nakazawa T, Doi $\mathrm{H}$, et al. Early high-dose intravenous methylprednisolone is effective in preserving retinal nerve fiber layer thickness in patients with neuromyelitis optica. Graefes Arch Clin Exp Ophthalmol. 2010 Dec;248(12):1777-1785. 
Figures

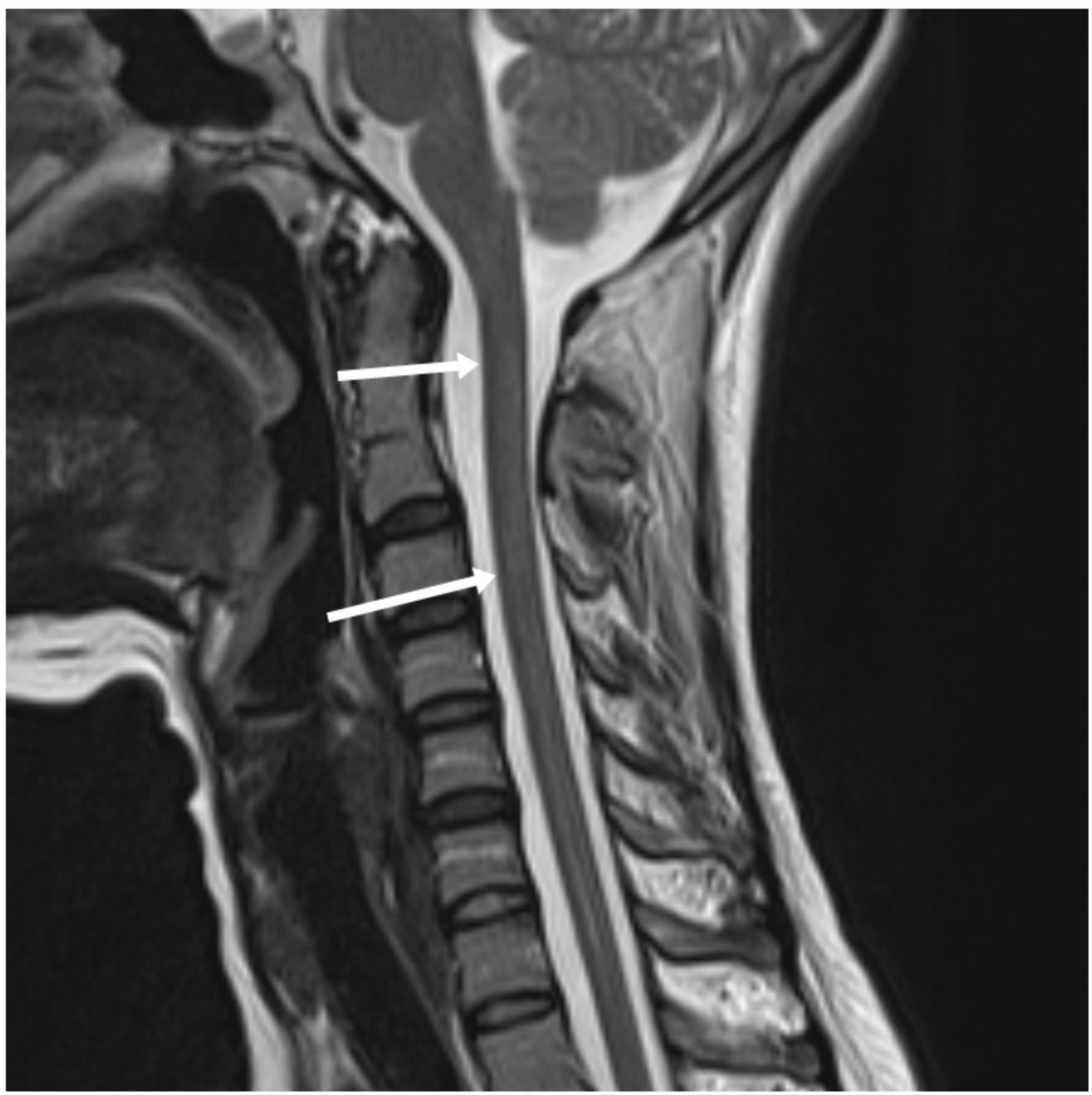

\section{Figure 1}

Weak hyperintense appearance in the form of plaques in T2-weighted series at the level of C2-C4 vertebrae in the cervical spinal cord 


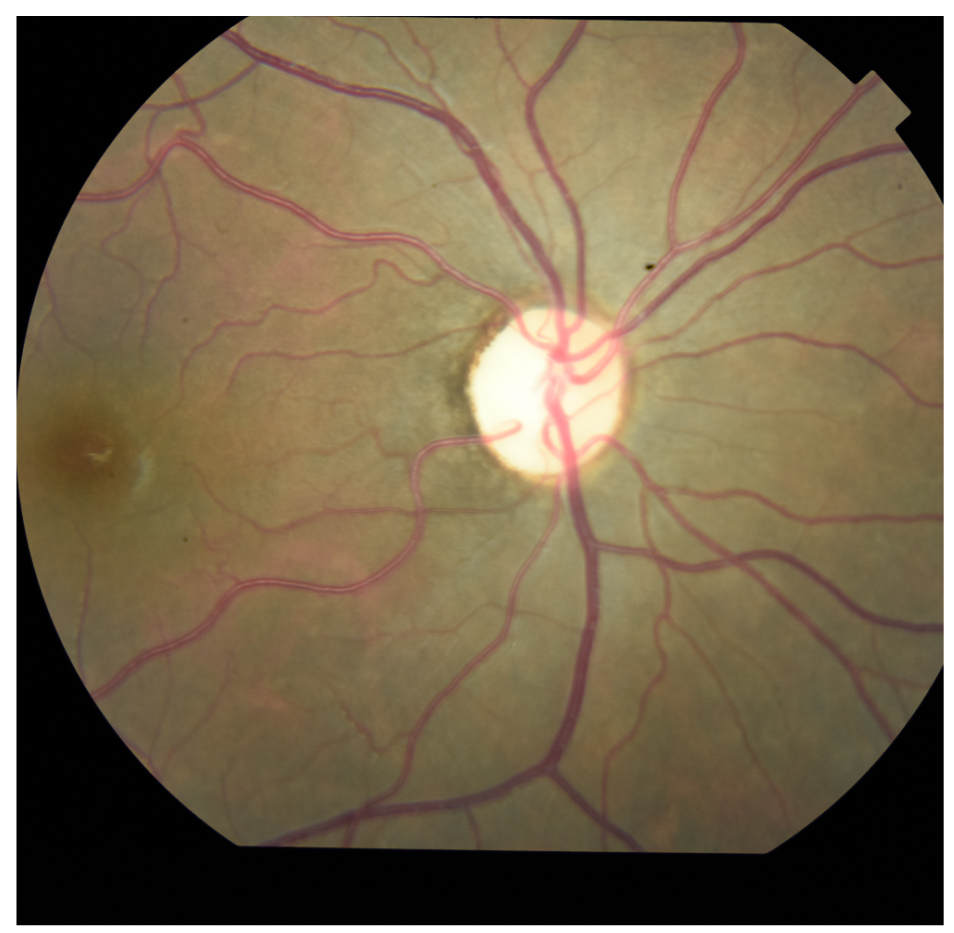

3D Disc

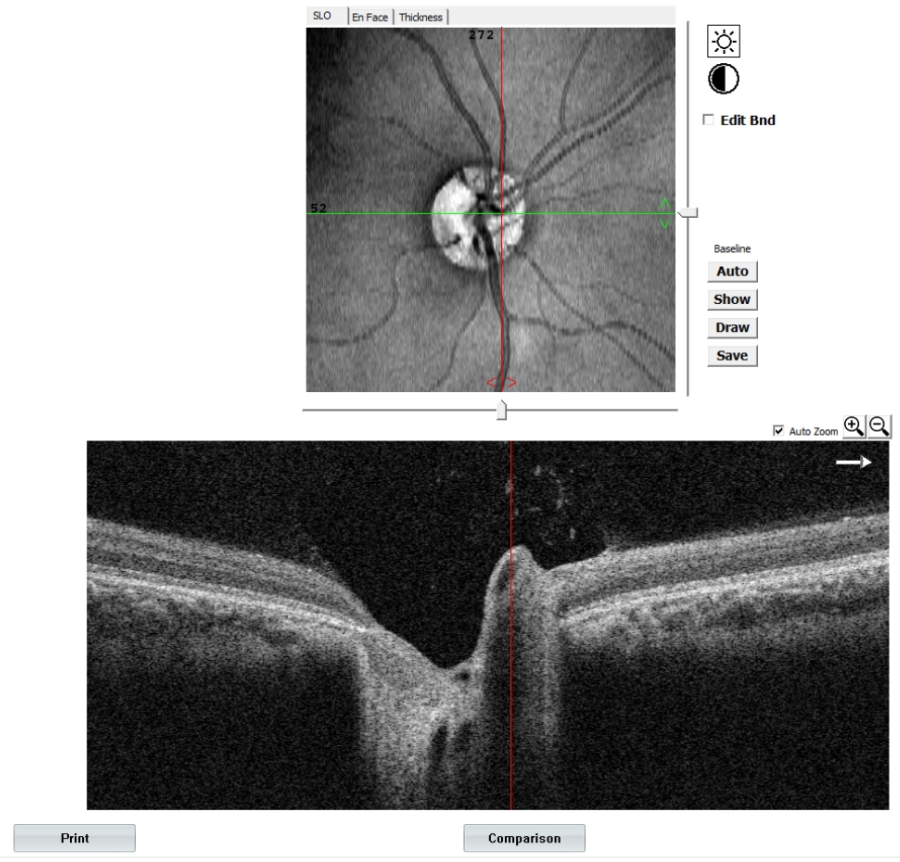

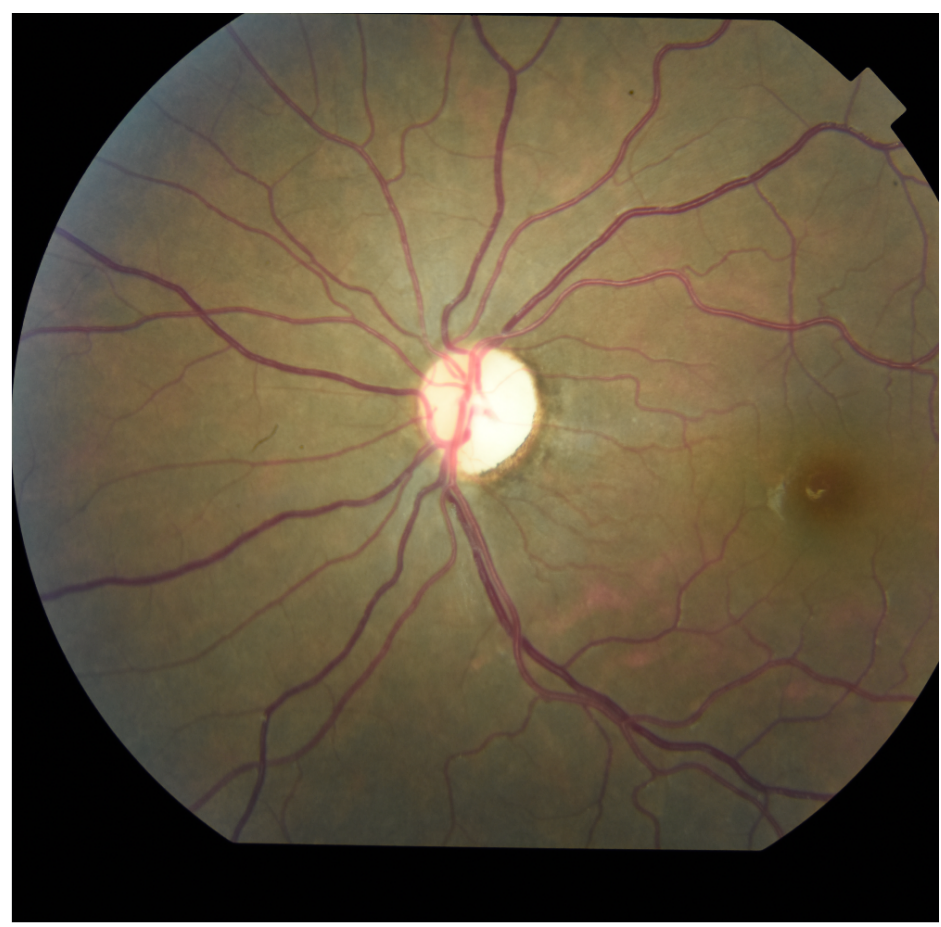

I Disc

Signal Stength Index

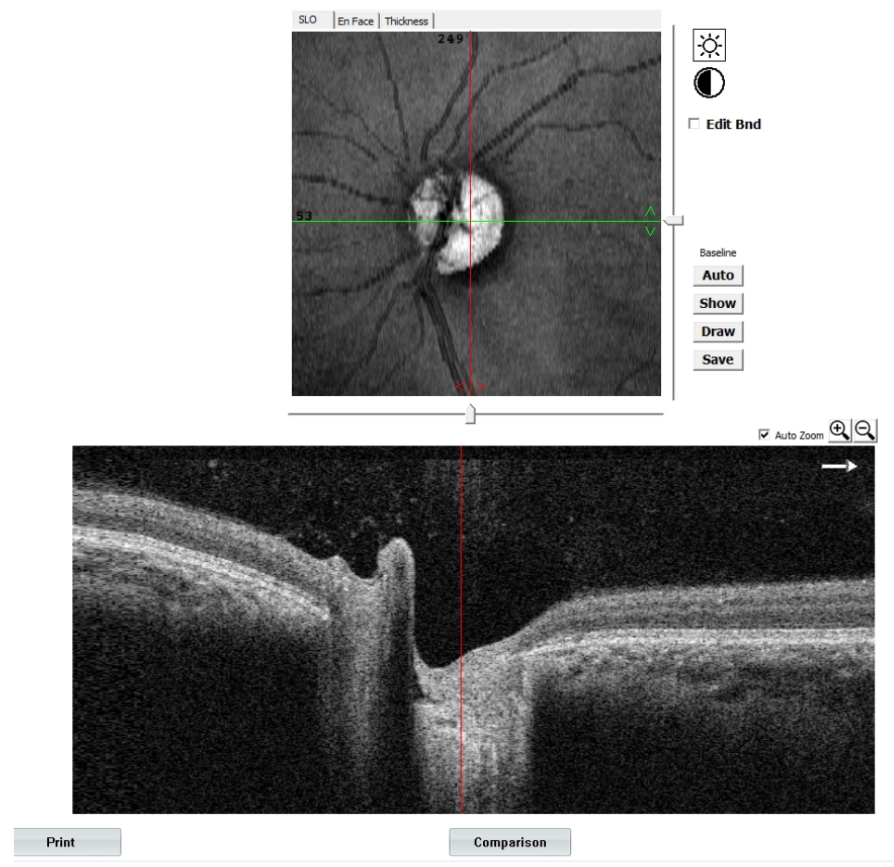

Figure 2

Right (a) and left (b) eye color fundus photograph and 3D optic disc sections of the case 